\title{
ON PRINCIPAL SECTIONS OF A PAIR OF FORMS
}

\author{
CHE-MAN CHENG
}

(Communicated by Lance W. Small)

\begin{abstract}
Let $H$ and $C$ be $n \times n$ Hermitian matrices with $C$ positive definite. Let $H\left(i_{1}, \ldots, i_{r}\right)$ denote the submatrix of $H$ formed by deleting the rows and columns $i_{1}, \ldots, i_{r}$ of $H$. In this paper, with $r_{1}+\cdots+r_{k} \leq n$, we study the roots of the determinantal equation $\operatorname{det}(\lambda C-H)=0$ and those of

$$
\operatorname{det}\left((\lambda C-H)\left(r_{1}+\cdots+r_{i-1}+1, \ldots, r_{1}+\cdots+r_{i}\right)\right)=0
$$

for $i=1, \ldots, k$.
\end{abstract}

\section{INTRODUCTION}

Let $\mathscr{M}_{n}$ denote the set of all $n \times n$ complex matrices. For $A \in \mathscr{M}_{n}$, let $A\left(i_{1}, \ldots, i_{r}\right)$ denote the submatrix of $A$ formed by deleting the rows and columns $i_{1}, \ldots, i_{r}$ of $A$. Concerning the eigenvalues of a Hermitian matrix and those of its principal submatrices, we have the following well-known interlacing theorem (e.g. [2] or [3, pp. 185-188]).

Theorem A. Let $H$ be an $n \times n$ Hermitian matrix with eigenvalues $\lambda_{1} \leq \cdots \leq \lambda_{n}$ and $1 \leq r \leq n-1$ be fixed. Then there exists a unitary matrix $U$ such that $U H U^{*}(1, \ldots, r)$ has eigenvalues $\eta_{1} \leq \cdots \leq \eta_{n-r}$ if and only if

$$
\lambda_{j} \leq \eta_{j} \leq \lambda_{j+r}, \quad j=1, \ldots, n-r .
$$

The eigenvalues of $H$ are the roots of the equation $\operatorname{det}(\lambda I-H)=0$. A more general setting is to consider the roots of

$$
\operatorname{det}(\lambda C-H)=0
$$

where $C$ is positive definite. It is known (see [6] or Theorem 1 in Section 2) that there is a Hermitian $H$ such that the roots of (2) are $\lambda_{1} \leq \cdots \leq \lambda_{n}$ and the roots of

$$
\operatorname{det}((\lambda C-H)(1, \ldots, r))=0
$$

are $\eta_{1} \leq \cdots \leq \eta_{n-r}$ if and only if (1) is satisfied.

Instead of just one, let us consider several principal submatrices at the same time. In [5], Thompson proved the result below when $r_{1}=\cdots=r_{k}=1$. It was generalized to the case when $r_{1}=\cdots=r_{k}$ in [1], but actually the proof there can be modified so as to obtain the following theorem.

Received by the editors February 24, 1994.

1991 Mathematics Subject Classification. Primary 15A42.

(C)1995 American Mathematical Society 
Theorem B. Let $H$ be an $n \times n$ Hermitian matrix with eigenvalues $\lambda_{1} \leq \cdots \leq$ $\lambda_{n}$, and $r_{1}, \ldots, r_{k}(k \geq 2)$ be positive integers such that $r_{1}+\cdots+r_{k} \leq n$. Then as $U$ varies over all unitary matrices, the eigenvalues $\eta_{1}^{(i)} \leq \cdots \leq \eta_{n-r_{i}}^{(i)}$ of the principal submatrix $U H U^{*}\left(r_{1}+\cdots+r_{i-1}+1, \ldots, r_{1}+\cdots+r_{i}\right)$ of $U H U^{*}$, for $1 \leq i \leq k$, independently assume all values permitted by the interlacing inequalities

$$
\lambda_{j} \leq \eta_{j}^{(i)} \leq \lambda_{j+r_{i}}, \quad j=1, \ldots, n-r_{i}, \quad i=1, \ldots, k,
$$

if and only if each distinct eigenvalue of $H$ has multiplicity at least $r_{1}+\cdots+r_{k}$.

In this paper, we consider the parallel problem for the pencil $\lambda C-H$. The result when $r_{1}=\cdots=r_{k}=1$ is given in [6]. Here, making use of Theorem B, we solve the problem for the general case (see Theorem 2 in Section 2).

In our discussion, let $I$ and $O$ be, respectively, the identity and zero matrices of appropriate order. The direct sum of matrices $A$ and $B$ is denoted by $A \oplus B$, and let $\operatorname{diag}\left(\lambda_{1}, \ldots, \lambda_{n}\right)$ be the diagonal matrix with diagonal entries $\lambda_{1}, \ldots, \lambda_{n}$. Let $\langle$,$\rangle denote the Euclidean inner product on \mathbb{C}^{n}$.

\section{RESULTS}

Throughout this section, let $C$ be an $n \times n$ positive definite matrix and suppose $C=X X^{*}$ where $X$ is invertible. As the roots of (2) are exactly the eigenvalues of $X^{-1} H X^{*-1}$, we easily obtain the following lemma.

Lemma 1. For Hermitian $H$, (2) has roots $\lambda_{1}, \ldots, \lambda_{n}$ if and only if $H=$ $X U \operatorname{diag}\left(\lambda_{1}, \ldots, \lambda_{n}\right) U^{*} X^{*}$ for some unitary $U$.

For the sake of completeness, we prove the following theorem.

Theorem 1. There is an $n \times n$ Hermitian matrix $H$ such that (2) has roots $\lambda_{1} \leq \cdots \leq \lambda_{n}$ and (3) has roots $\eta_{1} \leq \cdots \leq \eta_{n-r}$ if and only if (1) holds.

Proof. By the Gram-Schmidt process, we can find an upper triangular matrix $T$ such that $X^{-1} T=W^{*}$ where $W$ is unitary. Then $X=T W$. Let $T=\left[\begin{array}{cc}T_{11} & T_{12} \\ O & T_{22}\end{array}\right]$ where $T_{11} \in \mathscr{M}_{r}, T_{22} \in \mathscr{M}_{n-r}$. For any

$$
H=X U \operatorname{diag}\left(\lambda_{1}, \ldots, \lambda_{n}\right) U^{*} X^{*}
$$

with $U$ unitary, we have

$$
\begin{aligned}
& (\lambda C-H)(1, \ldots, r) \\
& \quad=T_{22}\left(\lambda I-W U \operatorname{diag}\left(\lambda_{1}, \ldots, \lambda_{n}\right) U^{*} W^{*}(1, \ldots, r)\right) T_{22}^{*} .
\end{aligned}
$$

Hence, we know that the roots of (3) are exactly the eigenvalues of

$$
W U \operatorname{diag}\left(\lambda_{1}, \ldots, \lambda_{n}\right) U^{*} W^{*}(1, \ldots, r) .
$$

The result follows from Lemma 1 and Theorem A.

By Lemma 1, we easily obtain the following lemma.

Lemma 2. Let $H$ be an $n \times n$ Hermitian matrix, and suppose $\lambda_{0}$ is a root of (2) with multiplicity $m$. Then $\operatorname{rank}\left(\lambda_{0} C-H\right)=n-m$.

The following lemma can be deduced from [4, Lemma 4.1]. 
Lemma 3. Let $H$ be an $n \times n$ Hermitian matrix with eigenvalues $\lambda_{1} \leq \cdots \leq \lambda_{n}$. If $H(1, \ldots, r)$ has eigenvalues $\lambda_{r+1}, \ldots, \lambda_{n}$, then $H=H_{1} \oplus H_{2}$ where $H_{1} \in$ $\mathscr{M}_{r}$ has eigenvalues $\lambda_{1}, \ldots, \lambda_{r}$, and $H_{2} \in \mathscr{M}_{n-r}$ has eigenvalues $\lambda_{r+1}, \ldots, \lambda_{n}$.

Let $x_{j}$ denote the $j$-th column of $X^{-1}$, i.e. $X^{-1}=\left[x_{1} \vdots \ldots \vdots x_{n}\right]$.

Lemma 4. Let $r_{1}+r_{2} \leq n$. If $\lambda_{1} \leq \cdots \leq \lambda_{n}$ are real numbers with $\lambda_{1}<\lambda_{n}$ such that for $i=1,2$, and for any choice of $\eta_{1}^{(i)} \leq \cdots \leq \eta_{n-r_{i}}^{(i)}$ satisfying

$$
\lambda_{j} \leq \eta_{j}^{(i)} \leq \lambda_{j+r_{i}}, \quad j=1, \ldots, n-r_{i},
$$

there is an $n \times n$ Hermitian matrix $H$ such that (2) has roots $\lambda_{1}, \ldots, \lambda_{n}$ and

$$
\operatorname{det}\left((\lambda C-H)\left(r_{i-1}+1, \ldots, r_{i-1}+r_{i}\right)\right)=0
$$

has roots $\eta_{1}^{(i)}, \ldots, \eta_{n-r_{i}}^{(i)}$, then

(a) $\lambda_{1}=\cdots=\lambda_{r_{1}+r_{2}}$,

(b) $\lambda_{n-r_{1}-r_{2}+1}=\cdots=\lambda_{n}$,

(c) $\left\langle x_{p}, x_{q}\right\rangle=0$ for $p=1, \ldots, r_{1}$, and $q=r_{1}+1, \ldots, r_{1}+r_{2}$.

Proof. We first derive some preliminary results. Without loss of generality, we assume $r_{1} \leq r_{2}$. Let $T$ be an upper triangular matrix such that $X^{-1} T=W^{*}$ where $W$ is unitary. Write

$$
T=\left[\begin{array}{ccc}
T_{11} & T_{12} & T_{13} \\
O & T_{22} & T_{23} \\
O & O & T_{33}
\end{array}\right]
$$

where $T_{11} \in \mathscr{M}_{r_{1}}, T_{22} \in \mathscr{M}_{r_{2}}$, and the $T_{i i}$ 's are invertible. By Lemma 1, for any Hermitian $H$ such that (2) has roots $\lambda_{1}, \ldots, \lambda_{n}$,

$$
\lambda C-H=T\left(\lambda I-W U \operatorname{diag}\left(\lambda_{1}, \ldots, \lambda_{n}\right) U^{*} W^{*}\right) T^{*}
$$

for some unitary $U$. Let $A=W U \operatorname{diag}\left(\lambda_{1}, \ldots, \lambda_{n}\right) U^{*} W^{*}$ so that it is a Hermitian matrix with eigenvalues $\lambda_{1}, \ldots, \lambda_{n}$, and

$$
\lambda C-H=T(\lambda I-A) T^{*} .
$$

Now suppose $H$ is chosen such that $\eta_{1}^{(1)}=\lambda_{r_{1}+1}, \ldots, \eta_{n-r_{1}}^{(1)}=\lambda_{n}$. From (7), and as in the proof of Theorem 1, we deduce that $\operatorname{det}\left(\lambda I-A\left(1, \ldots, r_{1}\right)\right)=0$ has roots $\lambda_{r_{1}+1}, \ldots, \lambda_{n}$, i.e. $A\left(1, \ldots, r_{1}\right)$ has eigenvalues $\lambda_{r_{1}+1}, \ldots, \lambda_{n}$. By Lemma 3, let

$$
A=\left[\begin{array}{ccc}
A_{11} & O & O \\
O & A_{22} & A_{23} \\
O & A_{32} & A_{33}
\end{array}\right]
$$

where $A_{11} \in \mathscr{M}_{r_{1}}$ has eigenvalues $\lambda_{1}, \ldots, \lambda_{r_{1}}, A_{22} \in \mathscr{M}_{r_{2}},\left[\begin{array}{c}A_{22} A_{23} \\ A_{33} A_{33}\end{array}\right]$ has eigenvalues $\lambda_{r_{1}+1}, \ldots, \lambda_{n}$. This is the case irrespective of the choice of $\eta_{1}^{(2)}, \ldots, \eta_{n-r_{2}}^{(2)}$. With the above $H$ (and hence $A$ ),

$$
\begin{aligned}
\operatorname{det}\left((\lambda C-H)\left(r_{1}+1, \ldots, r_{1}+r_{2}\right)\right) & \operatorname{det}\left(\left[\begin{array}{cc}
T_{11}\left(\lambda I-A_{11}\right) T_{11}^{*} & O \\
O & O
\end{array}\right]\right. \\
+ & {\left.\left[\begin{array}{cc}
T_{12} & T_{13} \\
O & T_{33}
\end{array}\right]\left(\lambda I-\left[\begin{array}{ll}
A_{22} & A_{23} \\
A_{32} & A_{33}
\end{array}\right]\right)\left[\begin{array}{cc}
T_{12}^{*} & O \\
T_{13}^{*} & T_{33}^{*}
\end{array}\right]\right) . }
\end{aligned}
$$


Let

$$
\begin{aligned}
f_{1}(\lambda) & =\left[\begin{array}{cc}
T_{11}\left(\lambda I-A_{11}\right) T_{11}^{*} & O \\
O & O
\end{array}\right] \\
f_{2}(\lambda) & =\left[\begin{array}{cc}
T_{12} & T_{13} \\
O & T_{33}
\end{array}\right]\left(\lambda I-\left[\begin{array}{ll}
A_{22} & A_{23} \\
A_{32} & A_{33}
\end{array}\right]\right)\left[\begin{array}{cc}
T_{12}^{*} & O \\
T_{13}^{*} & T_{33}^{*}
\end{array}\right] .
\end{aligned}
$$

(a) Suppose $H$ is chosen such that $\eta_{1}^{(2)}=\lambda_{r_{2}+1}, \ldots, \eta_{n-r_{2}}^{(2)}=\lambda_{n}$. Then $\lambda_{n}$ is a root of $\operatorname{det}\left(f_{1}(\lambda)+f_{2}(\lambda)\right)=0$. If $\lambda_{r_{1}}=\lambda_{n}$, then (as $\left.r_{1} \leq r_{2}\right) \eta_{1}^{(2)}=$ $\cdots=\eta_{n-r_{2}}^{(2)}=\lambda_{r_{1}}$ and so by Lemma $2 f_{1}\left(\lambda_{r_{1}}\right)+f_{2}\left(\lambda_{r_{1}}\right)=O$. Since $\left[\begin{array}{ll}A_{22} & A_{23} \\ A_{32} & A_{33}\end{array}\right]$ has eigenvalues $\lambda_{r_{1}+1}, \ldots, \lambda_{n}$, we see that $f_{2}\left(\lambda_{r_{1}}\right)=O$ and hence $f_{1}\left(\lambda_{r_{1}}\right)=O$. Consequently, as $T_{11}$ is invertible, $A_{11}=\lambda_{r_{1}} I$ and so $\lambda_{1}=\cdots=\lambda_{n}$. This contradicts $\lambda_{1}<\lambda_{n}$.

Now suppose $\lambda_{r_{1}}<\lambda_{n}$. Let $z=\left(z_{1}, z_{2}\right)$, where $z_{1} \in \mathbb{C}^{r_{1}}$ and $z_{2} \in \mathbb{C}^{n-r_{1}-r_{2}}$, be a nonzero vector such that

$$
z\left(f_{1}\left(\lambda_{n}\right)+f_{2}\left(\lambda_{n}\right)\right) z^{*}=0 .
$$

As $T_{11}\left(\lambda_{n} I-A_{11}\right) T_{11}^{*}$ is positive definite and $f_{2}\left(\lambda_{n}\right)$ is positive semidefinite, we deduce that $z_{1}=0$ and

$$
z_{2} T_{33}\left(\lambda_{n} I-A_{33}\right) T_{33}^{*} z_{2}^{*}=0 \text {. }
$$

If $r_{1}+r_{2}=n$ so that no $A_{33}$ appears, then $z=z_{1}$ and thus $z_{1}=0$ gives a contradition. We now assume $r_{1}+r_{2}<n$. As $\lambda_{n} I-A_{33}$ is positive semidefinite and $T_{33}$ is invertible, we conclude that $\lambda_{n}$ is an eigenvalue of $A_{33}$. If $\lambda_{r_{1}}<\lambda_{n-1}$, let $V$ be a unitary matrix such that $V A_{33} V^{*}=\operatorname{diag}\left(\alpha_{1}, \ldots, \alpha_{n-r_{1}-r_{2}-1}, \lambda_{n}\right)$. Then by Lemma 3

$$
(I \oplus V)\left[\begin{array}{ll}
A_{22} & A_{23} \\
A_{32} & A_{33}
\end{array}\right](I \oplus V)^{*}=\left[\begin{array}{ccc}
A_{22} & \tilde{A}_{23} & O \\
\tilde{A}_{32} & D & O \\
O & O & \lambda_{n}
\end{array}\right]
$$

where

$$
D=\operatorname{diag}\left(\alpha_{1}, \ldots, \alpha_{n-r_{1}-r_{2}-1}\right)
$$

By multiplying on the left a suitable matrix of the form $\left[\begin{array}{ll}I & * \\ 0 & 1\end{array}\right]$ to the matrix $\left(I \oplus V T_{33}^{-1}\right)\left(f_{1}(\lambda)+f_{2}(\lambda)\right)\left(I \oplus V T_{33}^{-1}\right)^{*}$, we see that

$$
\begin{aligned}
& \operatorname{det}\left\{\left[\begin{array}{cc}
T_{11}\left(\lambda I-A_{11}\right) T_{11}^{*} & O \\
O & O
\end{array}\right]\right. \\
& \left.\quad+\left[\begin{array}{cc}
T_{12} & \widetilde{T}_{13} \\
O & I
\end{array}\right]\left(\lambda I-\left[\begin{array}{cc}
A_{22} & \tilde{A}_{23} \\
\tilde{A}_{32} & D
\end{array}\right]\right)\left[\begin{array}{cc}
T_{12}^{*} & O \\
\tilde{T}_{13}^{*} & I
\end{array}\right]\right\}=0
\end{aligned}
$$

has root $\lambda_{r_{1}+1}, \ldots, \lambda_{n-1}$. Here, $\widetilde{T}_{13}$ is the matrix obtained by deleting the last column of $T_{13} V^{*}$. Repeating the same argument as above, we see that $\lambda_{n-1}=\alpha_{j}$ for some $j$. Inductively, we conclude that if $\lambda_{r_{1}}<\lambda_{\ell}$, then $\lambda_{\ell}$ is an eigenvalue of $A_{33}$ counting multiplicity. Consequently, we deduce that $\lambda_{r_{1}}=\cdots=\lambda_{r_{1}+r_{2}}$. Furthermore, by Theorem $\mathrm{A}$, the eigenvalues of $A_{33}$ are the

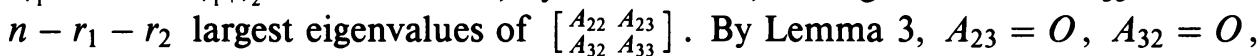
and $A_{22}=\lambda_{r_{1}} I$. Now suppose $\lambda_{r_{1}}=\cdots=\lambda_{r_{1}+r_{2}}=\cdots=\lambda_{s}<\lambda_{s+1}$ where 
$s \geq r_{1}+r_{2}$. Then, by Lemma $2, \operatorname{rank}\left(f_{1}\left(\lambda_{r_{1}}\right)+f_{2}\left(\lambda_{r_{1}}\right)\right)=n-s$. Now, as

$$
f_{1}\left(\lambda_{r_{1}}\right)+f_{2}\left(\lambda_{r_{1}}\right)=T_{11}\left(\lambda_{r_{1}} I-A_{11}\right) T_{11}^{*} \oplus T_{33}\left(\lambda_{r_{1}} I-A_{33}\right) T_{33}^{*}
$$

we conclude that $A_{11}=\lambda_{r_{1}} I$. Hence $\lambda_{1}=\cdots=\lambda_{r_{1}+r_{2}}$.

(b) If $\lambda_{1}, \ldots, \lambda_{n}$ satisfy the condition given in the hypothesis of the lemma, then so are $-\lambda_{n}, \ldots,-\lambda_{1}$ because one can take $-H$ instead of $H$ in the consideration of roots of (2) and (5). So, by part (a), we have $\lambda_{n-r_{1}-r_{2}+1}=$ $\cdots=\lambda_{n}$.

(c) It suffices to show $T_{12}$ given in (6) is the zero matrix. By (a) and (b), we now have $\lambda_{1}=\cdots=\lambda_{s}<\lambda_{s+1} \leq \cdots \leq \lambda_{n}$ where $r_{1}+r_{2} \leq s \leq n-r_{1}-r_{2}$. By Lemma 2 , if we choose $H$ such that $\eta_{1}^{(2)}=\lambda_{1}, \ldots, \lambda_{n-r_{2}}^{(2)}=\lambda_{n-r_{2}}$, then $\operatorname{rank}\left(f_{1}\left(\lambda_{1}\right)+f_{2}\left(\lambda_{1}\right)\right)=n-r_{2}-s$. Notice that now we have $f_{1}\left(\lambda_{1}\right)=O$. If $T_{12} \neq O$, then $\operatorname{rank}\left(\left[\begin{array}{cc}T_{12} & T_{13} \\ O & T_{33}\end{array}\right]\right)>n-r_{1}-r_{2}$ and so $\operatorname{rank}\left(f_{2}\left(\lambda_{1}\right)\right)>n-r_{1}-r_{2}-$ $\left(s-r_{1}\right)=n-s-r_{2}$. Thus we have a contradiction. Hence $T_{12}=O$.

Theorem 2. Let $C$ be positive definite, $\lambda_{1} \leq \cdots \leq \lambda_{n}$ be fixed real numbers with $\lambda_{1}<\lambda_{n}$, and $r_{1}, \ldots, r_{k}(k \geq 2)$ be positive integers such that $r_{1}+\cdots+r_{k} \leq n$. Then the following conditions (I) and (II) are equivalent.

(I) For any choice of $\eta_{1}^{(i)} \leq \cdots \leq \eta_{n-r_{i}}^{(i)}, i=1, \ldots, k$, satisfying (4) there is an $n \times n$ Hermitian $H$ such that the roots of (2) are $\lambda_{1}, \ldots, \lambda_{n}$ and, for $i=1, \ldots, k$, the roots of

$$
\operatorname{det}\left((\lambda C-H)\left(r_{1}+\cdots+r_{i-1}+1, \ldots, r_{1}+\cdots+r_{i}\right)\right)=0
$$

are $\eta_{1}^{(i)}, \ldots, \eta_{n-r_{i}}^{(i)}$.

(II) (i) Each distinct number among $\lambda_{1}, \ldots, \lambda_{n}$ has multiplicity at least $r_{1}+$ $\cdots+r_{k}$, and

(ii) if $C^{-1}=\left(M_{i j}\right)_{i, j=1, \ldots, k+1}$ with $M_{i i} \in \mathscr{M}_{r_{i}}, i=1, \ldots, k$, then $M_{i j}=O$ for $1 \leq i \neq j \leq k$.

Proof. Let $N_{1}=\left\{1, \ldots, r_{1}\right\}, \ldots, N_{k}=\left\{r_{1}+\cdots+r_{k-1}+1, \ldots, r_{1}+\cdots+r_{k}\right\}$. If we consider pairwise $N_{r}, N_{s}$ and $P C P^{*}$ for suitable permutation matrix $P$, then by Lemma 4 we can deduce that a necessary condition for (I) is that, with $X^{-1}=\left[x_{1} \vdots \cdots \vdots x_{n}\right]$,

$$
\left\langle x_{p}, x_{q}\right\rangle=0, \quad p \in N_{r}, \quad q \in N_{s}, \quad 1 \leq r \neq s \leq k .
$$

As $C^{-1}=X^{-1 *} X^{-1}$, this is equivalent to saying that $C^{-1}$ satisfies (II)(ii). Now suppose this condition holds. Then we can find an upper triangular

$$
T=\left[\begin{array}{cccc|c}
T_{11} & O & \cdots & O & \star \\
O & T_{22} & \cdots & O & \star \\
\vdots & \vdots & \ddots & \vdots & \\
O & O & \cdots & T_{k k} & \\
\hline O & O & \cdots & O & T_{k+1, k+1}
\end{array}\right]
$$


such that $X=T W$ where $W$ is unitary. Then for any

$$
H=X U \operatorname{diag}\left(\lambda_{1}, \ldots, \lambda_{n}\right) U^{*} X^{*}
$$

with $U$ unitary, and $1 \leq i \leq k$,

$$
\begin{aligned}
& \operatorname{det}\left((\lambda C-H)\left(r_{1}+\cdots+r_{i-1}+1, \ldots, r_{1}+\cdots+r_{i}\right)\right) \\
& =\left(\prod_{\substack{j=1 \\
j \neq i}}^{k+1} \operatorname{det} T_{j j} \operatorname{det} T_{j j}^{*}\right) \\
& \quad \times \operatorname{det}\left(\lambda I-W U \operatorname{diag}\left(\lambda_{1}, \ldots, \lambda_{n}\right)\right. \\
& \left.\quad \cdot U^{*} W^{*}\left(r_{1}+\cdots+r_{i-1}+1, \ldots, r_{1}+\cdots+r_{i}\right)\right) .
\end{aligned}
$$

That is, the roots of $(8)$ are exactly the eigenvalues of

$$
W U \operatorname{diag}\left(\lambda_{1}, \ldots, \lambda_{n}\right) U^{*} W^{*}\left(r_{1}+\cdots+r_{i-1}+1, \ldots, r_{1}+\cdots+r_{i}\right) \text {. }
$$

The result follows from Lemma 1 and Theorem $B$.

Finally, we state without proof the following theorem for completeness.

Theorem 3. The roots of (2) satisfy $\lambda_{1}=\cdots=\lambda_{n}=\alpha$ if and only if $H=\alpha C$. If this is so,

$$
\begin{aligned}
& \operatorname{det}\left((\lambda C-H)\left(r_{1}+\cdots+r_{i-1}+1, \ldots, r_{1}+\cdots+r_{i}\right)\right) \\
& \quad=\operatorname{det}\left(C\left(r_{1}+\cdots+r_{i-1}+1, \ldots, r_{1}+\cdots+r_{i}\right)\right)(\lambda-\alpha)^{n-r_{i}} .
\end{aligned}
$$

\section{ACKNOWLEDGMENT}

I would like to thank Professor R. C. Thompson for bringing my attention to [6] and suggesting the investigation of Theorem 2.

\section{REFERENCES}

1. C. M. Cheng, Independence of eigenvalues and independence of singular values of submatrices, Linear Algebra Appl. 166 (1992), 115-129.

2. K. Fan and G. Pall, Imbedding conditions for Hermitian and normal matrices, Canad. J. Math. 9 (1958), 298-304.

3. R. A. Hom and C. R. Johnson, Matrix analysis, Cambridge Univ. Press, New York, 1991.

4. C. K. Li, Matrices with some extremal properties, Linear Algebra Appl. 101 (1988), 255-267.

5. R.C. Thompson, Principal submatrices IV. On the independence of eigenvalues of different principal submatrices, Linear Algebra Appl. 2 (1969), 355-374.

6. R. C. Thompson, Principal submatrices VIII. Principal sections of a pair of forms, Rocky Mountain J. Math. 2 (1972), 97-110.

Faculty of Science and Technology, University of Macau, Macau

E-mail address: fstcmcQuealab.umac.mo 